\title{
Correspondence
}

\section{Partially Adaptive Beamformer Design Subject to Worst-Case Performance Constraints}

Feng Qian and Barry D. Van Veen

Abstract-This correspondence proposes two methods for designing partially adaptive beamformers that satisfy a performance specification over a set of likely interference scenarios. Both methods choose the adaptation space in a sequential fashion; the dimension is increased one by one until the performance specification is attained. In the multilevel point design method, each dimension of the adaptation space is chosen to give optimum performance at a single interference scenario. The constrained minimization design method chooses each dimension of the adaptation space to exactly satisfy the performance specification at a single interference scenario while approximately minimizing the average interference output power over neighboring scenarios. Simulations indicate that both methods result in better performance than existing methods while using fewer degrees of freedom.

\section{INTRODUCTION}

Partially adaptive beamformers employ only a portion of the available adaptive degrees of freedom to reduce the computational complexity of adaptive algorithms and improve the convergence rate [1], [4], [14]. On the negative side, the steady-state interference cancellation capability of a beamformer degrades as the number of adaptive weights are reduced. This degradation is strongly dependent on which adaptive degrees of freedom are utilized by the partially adaptive beamformer. The goal of partially adaptive beamformer design is to choose a low dimensional adaptation space that provides satisfactory steady state interference cancellation.

A variety of partially adaptive beamformer design procedures (see e.g., [1], [2], [4], [6], [8], [9], [11], [12]) have been proposed. Eigenstructure and beam based designs [2], [4], [9], [12] can result in excellent steady state interference cancellation but often require more adaptive weights than necessary [13]. Power minimization designs [11] attempt to minimize the average interference output power over a set of likely interference scenarios $\mathcal{Q}$ for a given adaptive dimension. Unfortunately, this optimization problem is intractable so a suboptimal solution is proposed wherein each component of the adaptation space is optimized separately over a distinct subset of $\mathcal{Q}$. Although good performance is obtained with relatively small numbers of adaptive weights, the procedure is not very systematic; in order to obtain the best performance, the subsets of $\mathcal{Q}$ used to design individual components must be selected through a trial and error process.

Here, the partially adaptive beamformer design problem is approached from an alternate perspective. The objective is to minimize the number of adaptive degrees of freedom subject to a constraint on the worst case interference cancellation performance loss over $\mathcal{Q}$.

Manuscript received May 20, 1992; revised June 28, 1993. This work was supported by the Aileen S. Andrew Foundation, the National Science Foundation under award MIP-8958559, and the Army Research Office under grant DAALO3-89-K-0141. The associate editor coordinating the review of this paper and approving it for publication was Prof. John A. Stuller.

The authors are with the Department of Electrical and Computer Engineering, University of Wisconsin, Madison, WI 53706.

IEEE Log Number 9216696.
Two systematic design procedures are developed. The multilevel point design method chooses each component of the adaptation space to achieve interference cancellation at a specific interference scenario equivalent to that obtained by a fully adaptive beamformer. The constrained minimization design method chooses each component of the adaptation space to exactly satisfy the worst case performance constraint at a specific interference scenario while approximately minimizing the average performance loss over neighboring scenarios. In both cases, the columns of the adaptation space are designed in a sequential manner, that is, the dimension of the adaptation space is increased one at a time until the performance constraint is satisfied over the entire set of possible interference scenarios. The interference scenario at which the design occurs is chosen by comparing the performance loss for the current adaptation space to a user specified threshold. If the loss exceeds the threshold, then a new dimension is added.

Simulations indicate that these approaches result in better interference cancellation performance with fewer adaptive degrees of freedom than the interference output power minimization method. Furthermore, no hand crafting is necessary; both of the proposed design methods are completely automated. We also note that these procedures are applicable to a broader class of problems than partially adaptive beamformer design. For example, they are applicable to subspace design for generalized likelihood ratio adaptive detection. In the adaptive detection problem, the subspace is chosen to maximize the signal to noise ratio over a set of possible interference scenarios. Maximization of the detector's SNR leads to a design problem of the same form as maximization of interference cancellation in a partially adaptive beamformer [3].

For notation, lower and upper case boldface symbols are used to represent vectors and matrices respectively, and superscript $H$ denotes complex conjugate transpose.

\section{Partially Adaptive Beamformer Design}

\section{A. Partially Adaptive Beamforming}

Assume that the desired signal is statistically uncorrelated with the interferers so the beamformer data covariance matrix is $\boldsymbol{R}_{x}=$ $\boldsymbol{R}_{s}+\boldsymbol{R}_{n}$, where $\boldsymbol{R}_{s}$ is the signal covariance matrix, and $\boldsymbol{R}_{n}$ is the interference and noise covariance matrix.

A generalized sidelobe canceller (GSC) implementation [5] of the linearly constrained minimum variance beamformer (see Fig. 1) decomposes the $n$-dimensional beamformer weight vector as

$$
\boldsymbol{w}=\boldsymbol{w}_{0}-\boldsymbol{T}_{n} \boldsymbol{w}_{n} .
$$

Here, $\boldsymbol{w}_{0} \in$ range $\left(\boldsymbol{R}_{s}\right)$ is a nonadaptive weight that provides a desired beamformer response to the signal and the $n \times p$ blocking matrix $\boldsymbol{T}_{n}$ satisfies $\boldsymbol{T}_{n}^{H} \boldsymbol{R}_{s}=O$. We refer to range $\left(T_{n}\right)$ as the adaptation space because $\boldsymbol{w}$ can only adapt the components that lie in this space. $w_{n}$ is a $p$-dimensional adaptive weight that is chosen to minimize the beamformer output power, i.e.,

$$
\min _{\boldsymbol{w}_{n}}\left(\boldsymbol{w}_{0}-\boldsymbol{T}_{n} \boldsymbol{w}_{n}\right)^{H} \boldsymbol{R}_{x}\left(\boldsymbol{w}_{0}-\boldsymbol{T}_{n} \boldsymbol{w}_{n}\right) .
$$

The optimal adaptive weight vector is easily found as

$$
\boldsymbol{w}_{n}=\left(\boldsymbol{T}_{n}^{H} \boldsymbol{R}_{n} \boldsymbol{T}_{n}\right)^{-1} \boldsymbol{T}_{n}^{H} \boldsymbol{R}_{n} \boldsymbol{w}_{0}
$$




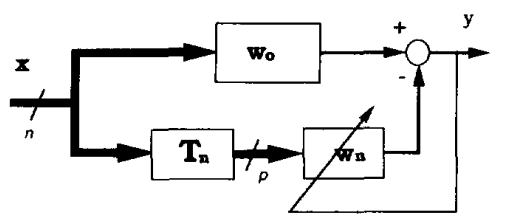

Fig. 1. GSC beamformer implementation.

and the corresponding minimum output power is given by

$$
P_{0}\left(T_{n}\right)=P_{s}+P_{n}-P_{n c}\left(T_{n}\right)
$$

where

$$
\begin{aligned}
P_{s} & =w_{0}^{H} \boldsymbol{R}_{s} w_{0} \\
P_{n} & =w_{0}^{H} R_{n} w_{0} \\
P_{n c}\left(T_{n}\right) & =w_{0}^{H} R_{n} T_{n}\left(T_{n}^{H} R_{n} T_{n}\right)^{-1} T_{n}^{H} R_{n} w_{0} .
\end{aligned}
$$

Note that $P_{n c}$ is the only term in (4) dependent on $\boldsymbol{T}_{n}$.

For a fully adaptive beamformer, the $n \times q(q>p)$ blocking matrix $C_{n}$ satisfies $\operatorname{range}\left(C_{n}\right)=\operatorname{range}^{\perp}\left(\boldsymbol{R}_{s}\right)$. Therefore, $\operatorname{range}\left(T_{n}\right) \subset$ range $\left(C_{n}\right)$. It can be shown that $P_{n c}\left(T_{n}\right) \leq P_{n c}\left(C_{n}\right)=P_{n c}^{\max }$ with equality if range $\left(T_{n}\right)=\operatorname{range}\left(C_{n}\right)$ [11]. However, this is not the only case for which equality holds. In fact, for any given interference scenario, the 1-D adaptation space represented by

$$
\boldsymbol{t}_{n}=\boldsymbol{C}_{n}\left(C_{n}^{H} \boldsymbol{R}_{n} C_{n}\right)^{-1} C_{n}^{H} \boldsymbol{R}_{n} \boldsymbol{w}_{0}
$$

achieves the maximum level of interference cancellation, i.e., $P_{n c}\left(t_{n}\right)=P_{n c}^{\max }$. In practice, $\boldsymbol{R}_{n}$ is usually unknown. Thus, the optimal 1-D adaptation space is also unknown and multiple degrees of freedom are usually employed to obtain good performance.

\section{B. Partially Adaptive Beamformer Performance Criterion}

The performance of a partially adaptive beamformer is evaluated in terms of its interference output power relative to that of the fully adaptive beamformer for a set of likely interference scenarios $\mathcal{Q}=$ $\left\{\Theta_{k}, k=1,2, \cdots, K\right\}$. For example, the parameter $\Theta$ can represent the number of interferers, their locations, spectral characteristics, etc. We assume that $\boldsymbol{R}_{n}$ is completely determined by $\Theta$ with the explicit dependence indicated by $\boldsymbol{R}_{n}(\Theta)$. The partially adaptive beamformer interference and noise output power is given by

$$
P_{i}\left(T_{n}, \Theta\right)=P_{n}(\Theta)-P_{n c}\left(T_{n}, \Theta\right) \text {. }
$$

Denote the interference and noise output power of the fully adaptive beamformer as

$$
P_{i}^{\min }(\Theta)=P_{n}(\Theta)-P_{n c}^{\max }(\Theta)
$$

and define the performance index as

$$
I\left(\boldsymbol{T}_{n}, \Theta\right)=\frac{P_{i}^{\min }(\Theta)}{P_{i}\left(\boldsymbol{T}_{n}, \Theta\right)}
$$

Here, $I([], \theta)=P_{n}(\theta)-P_{n c}^{\max }(\theta) / P_{n}(\theta)$ is the performance index of the correspoding nonadaptive beamformer. Note that
$I([], \theta) \leq I\left(T_{n}, \theta\right) \leq 1$. For design of $T_{n}$, we propose requiring that the performance index exceed a user-specified tolerance $\delta_{0}(\boldsymbol{\theta})$, that is

$$
\boldsymbol{I}\left(\boldsymbol{T}_{n}, \boldsymbol{\theta}\right) \geq \delta_{0}(\boldsymbol{\theta}), \quad \forall \boldsymbol{\theta} \in \mathcal{Q}
$$

Clearly, $\delta_{0}(\boldsymbol{\theta})$ must satisfy $\delta_{0}(\boldsymbol{\theta}) \leq 1$. For the remainder of the correspondence, we assume $\delta_{0}$ is independent of $\Theta$. For example, if $\delta_{0}=0.5$, then $P_{i}\left(T_{n}, \theta\right)$ is required to be within $3 \mathrm{~dB}$ of $P_{i}^{\mathrm{min}}(\theta)$.

\section{Partially Adaptive Beamformer Design Procedures}

Here, we provide two design methods for designing partially adaptive beamformers that satisfy the criterion (12). Both methods choose the columns of $T_{n}$ in a sequential manner. The sequential nature of the design is well described in terms of the modular adaptive beamforming structure introduced in [10]. Let $T_{n 0}$ be the current adaptation space and $t_{n}$ the additional column to be designed, i.e., $\boldsymbol{T}_{n}=\left[\begin{array}{ll}\boldsymbol{T}_{n 0} & \boldsymbol{t}_{n}\end{array}\right]$. If the existing adaptation space range $\left(\boldsymbol{T}_{n 0}\right)$ does not satisfy the performance specification, i.e., $P_{i}\left(T_{n 0}, \theta\right)>$ $P_{i}^{\text {min }}(\boldsymbol{\theta}) / \delta_{0}$, then $t_{n}$ is utilized to improve performance. It can be shown [7] that

$$
\begin{aligned}
P_{i}\left(\left[\begin{array}{ll}
\boldsymbol{T}_{n 0} & \boldsymbol{t}_{n}
\end{array}\right], \boldsymbol{\theta}\right)= & \boldsymbol{w}_{0}^{H} \boldsymbol{R}_{m}(\boldsymbol{\theta}) \boldsymbol{w}_{0} \\
& -\frac{\boldsymbol{w}_{0}^{H} \boldsymbol{R}_{m}(\Theta) \boldsymbol{t}_{n} \boldsymbol{t}_{n}^{H} \boldsymbol{R}_{m}(\boldsymbol{\theta}) \boldsymbol{w}_{0}}{\boldsymbol{t}_{n}^{H} \boldsymbol{R}_{m}(\Theta) \boldsymbol{t}_{n}}
\end{aligned}
$$

where

$$
\boldsymbol{R}_{m}(\boldsymbol{\theta})=\boldsymbol{R}_{n}(\boldsymbol{\theta})-\boldsymbol{R}_{n}(\boldsymbol{\theta}) \boldsymbol{T}_{n 0}\left(\boldsymbol{T}_{n 0}^{H} \boldsymbol{R}_{n}(\boldsymbol{\theta}) \boldsymbol{T}_{n 0}\right)^{-1} \boldsymbol{T}_{n 0}^{H} \boldsymbol{R}_{n}(\boldsymbol{\theta})
$$

The first term in (13) is $\boldsymbol{P}_{i}\left(\left[\boldsymbol{T}_{n 0}\right], \boldsymbol{\theta}\right)$; the second is the interference power reduction obtained by adding $t_{n}$.

The design process consists of adding columns to $T_{n}$ until (12) is satisfied for all interference scenarios in $\mathcal{Q}$. Each additional column will generally improve the performance for all interference scenarios even though it is designed to satisfy the performance specification at a single scenario. Consequently, we generally require far fewer columns in $\boldsymbol{T}_{\boldsymbol{n}}$ than the number of interference scenarios $K$. Note that the resulting adaptation space depends on the order in which the scenarios in $\mathcal{Q}$ are tested in the design. To make efficient use of the adaptive weights, the design process should initially focus on scenarios with the greatest need of performance improvement. This is approximately accomplished by defining a sequence of $L$ increasing performance levels $\delta_{1}<\delta_{2}<\cdots<\delta_{L}=\delta_{0}$. Let $T_{n 0}$ be the current adaptation space. At each performance level, we add columns to $\boldsymbol{T}_{n 0}$ until the current performance specification is satisfied. The pseudo-code at the bottom of this page describes this approach.

Two methods for designing $\boldsymbol{t}_{n}$ that guarantee satisfaction of the performance specification at a scenario $\boldsymbol{\theta}_{k}$ are now provided: a point design (PD) method and a constrained minimization design (CMD) method. First the components of $t_{n}$ that are not already represented in $\boldsymbol{T}_{n 0}$ are isolated by writing $\boldsymbol{t}_{n}=\boldsymbol{U}_{n} \boldsymbol{t}$ with $\boldsymbol{U}_{n}$ defined so that $\operatorname{range}\left(U_{n}\right) \oplus \operatorname{range}\left(\boldsymbol{T}_{n 0}\right)=\operatorname{range}\left(\boldsymbol{C}_{n}\right)$. Here, $\oplus$ represents the direct sum of subspaces. The problem is now to find an effective $t$.

The PD method chooses $\boldsymbol{t}$ such that the corresponding $\boldsymbol{t}_{n}$ provides maximum interference cancellation at a scenario $\theta_{k}$, i.e.,

$$
P_{i}\left(\left[\begin{array}{ll}
\boldsymbol{T}_{n 0} & \boldsymbol{t}_{n}
\end{array}\right], \Theta_{k}\right)=P_{i}^{\min }\left(\Theta_{k}\right)
$$

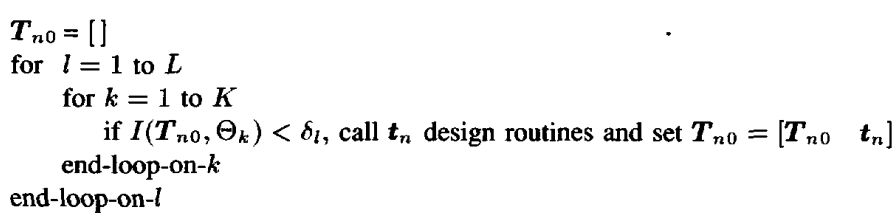

$\boldsymbol{T}_{n 0}=$ []

for $k=1$ to $K$ end-loop-on- $k$

end-loop-on- $l$ 
It can be shown that the PD solution is given by

$$
\boldsymbol{t}=\left(\boldsymbol{U}_{n}^{H} \boldsymbol{R}_{m}\left(\boldsymbol{\Theta}_{k}\right) \boldsymbol{U}_{n}\right)^{-1} \boldsymbol{U}_{n}^{H} \boldsymbol{R}_{m}\left(\boldsymbol{\Theta}_{k}\right) \boldsymbol{w}_{0} .
$$

Although the PD method is very simple, the performance generally exceeds the specification at $\boldsymbol{\theta}_{k}$ since the performance loss is forced to zero. In principle, this suggests that the number of adaptive weights obtained using the PD method is larger than necessary since the most efficient design should exactly meet (not exceed) the performance specification.

The goal of the CMD method is to further reduce the number of adaptive weights required to achieve the performance specification. At each level $\delta_{l}, \boldsymbol{t}_{n}$ is constrained to exactly satisfy the performance specification at the design point, i.e., $\left.P_{i}\left[\begin{array}{ll}\boldsymbol{T}_{n 0} & \boldsymbol{t}_{n}\end{array}\right], \boldsymbol{\theta}_{k}\right)=$ $P_{i}^{\text {min }}\left(\Theta_{k}\right) / \delta_{l}$. Unless $\delta_{l}=1$, the solution to this problem is not unique. The freedom is used here to approximately minimize the average interference output power in the neighborhood of $\boldsymbol{\theta}_{k}$. Let $\mathcal{Q}_{k}=\left\{\Theta_{k j}, j=1,2, \cdots J\right\}$ represent interference scenarios in the neighborhood of $\boldsymbol{\theta}_{k}$. The average interference output power over this neighborhood is expressed as

$$
\begin{aligned}
P_{i}^{a v}\left(\left[\begin{array}{ll}
\boldsymbol{T}_{n 0} & \boldsymbol{t}_{n}
\end{array}\right]\right)= & \sum_{j=1}^{J}\left(\boldsymbol{w}_{0}-\boldsymbol{w}_{k j} \boldsymbol{U}_{n} t\right)^{H} \\
& \cdot \boldsymbol{R}_{m}\left(\Theta_{k j}\right)\left(\boldsymbol{w}_{0}-w_{k j} \boldsymbol{U}_{n} \boldsymbol{t}\right)
\end{aligned}
$$

where $w_{k j}=\boldsymbol{t}^{H} \boldsymbol{U}_{n}^{H} \boldsymbol{R}_{m}\left(\Theta_{k j}\right) \boldsymbol{w}_{0} / \boldsymbol{t}^{H} \boldsymbol{U}_{n}^{H} \boldsymbol{R}_{m}\left(\boldsymbol{\theta}_{k j}\right) \boldsymbol{U}_{n} \boldsymbol{t}$. Unfortunately, the dependence of the weight $w_{k j}$ on $\boldsymbol{\theta}_{k j}$ and $t$ results in an analytically intractible optimization problem.

An approximation is now introduced to simplify the problem. We assume that the neighborhood $\mathcal{Q}_{k}$ is sufficiently small that $w_{k j}(j=$ $1,2, \cdots, J)$ is constant and equal to $w_{k}$. Since the magnitude of $t$ is arbitrary, we take $w_{k j}=w_{k}=1, j=1,2, \cdots, J$. The constrained minimization design criterion is now expressed as

$$
\begin{aligned}
\min _{\boldsymbol{t}} & \left(\boldsymbol{w}_{0}-\boldsymbol{U}_{n} \boldsymbol{t}\right)^{H} \boldsymbol{R}_{k}^{a v}\left(\boldsymbol{w}_{0}-\boldsymbol{U}_{n} \boldsymbol{t}\right) \\
\text { subject to } & \left(\boldsymbol{w}_{0}-\boldsymbol{U}_{n} \boldsymbol{t}\right)^{H} \boldsymbol{R}_{m}\left(\boldsymbol{\theta}_{k}\right)\left(\boldsymbol{w}_{0}-\boldsymbol{U}_{\mathrm{n}} \boldsymbol{t}\right) \leq \frac{P_{i}^{\min }\left(\boldsymbol{\theta}_{k}\right)}{\delta_{l}} .
\end{aligned}
$$

Here, $\boldsymbol{R}_{k}^{a v}=\Sigma_{j=1}^{J} \boldsymbol{R}_{m}\left(\Theta_{k j}\right)$. Note that the approximation does not affect the constraint. The solution to (17) is obtained using Lagrange multiplier techniques and can be expressed as follows. If the solution

$$
\boldsymbol{t}=\left(\boldsymbol{U}_{n}^{H} \boldsymbol{R}_{k}^{a v} \boldsymbol{U}_{n}\right)^{-1} \boldsymbol{U}_{n}^{H} \boldsymbol{R}_{k}^{a v} \boldsymbol{w}_{0}
$$

satisfies $I\left(\left[\begin{array}{ll}\boldsymbol{T}_{n 0} & \boldsymbol{U}_{n} t\end{array}\right], \boldsymbol{\theta}_{k}\right) \geq \delta_{l}$, then the constraint in (17) is inactive and $t_{n}=U_{n} t$ is chosen as the new component of the adaptation space. Otherwise, set

$$
\begin{aligned}
\boldsymbol{t}(\lambda)= & \left(\boldsymbol{U}_{n}^{H} \boldsymbol{R}_{k}^{a v} \boldsymbol{U}_{n}+\lambda \boldsymbol{U}_{n}^{H} \boldsymbol{R}_{m}\left(\boldsymbol{\Theta}_{k}\right) \boldsymbol{U}_{n}\right)^{-1} \\
& \cdot \boldsymbol{U}_{n}^{H}\left(\boldsymbol{R}_{k}^{a v}+\lambda \boldsymbol{R}_{m}\left(\boldsymbol{\Theta}_{k}\right)\right) \boldsymbol{w}_{0}
\end{aligned}
$$

where the Lagrange multiplier $\lambda$ is chosen to satisfy

$$
\left(\boldsymbol{w}_{0}-\boldsymbol{U}_{n} \boldsymbol{t}(\lambda)\right)^{H} \boldsymbol{R}_{m}\left(\boldsymbol{\theta}_{k}\right)\left(\boldsymbol{w}_{0}-\boldsymbol{U}_{n} \boldsymbol{t}(\lambda)\right)=\frac{P_{i}^{\min }\left(\boldsymbol{\theta}_{k}\right)}{\delta_{l}} .
$$

The new component of the adaptation space is then constructed as $\boldsymbol{t}_{n}=\boldsymbol{U}_{n} \boldsymbol{t}(\lambda)$.

Remark 1: In principle, choosing large neighborhoods $\mathcal{Q}_{k}$ should result in fewer adaptive weights for a given performance specification. However, the approximation used to derive (17) is only reasonable for small neighborhoods.

Remark 2: Our experience indicates that good results are usually achieved for both PD and CMD methods with $L=3$ or $L=4$ levels chosen to span a wide range of performance losses.
Remark 3: These multi-level design procedures order the columns of $\boldsymbol{T}_{n}$ according to their importance for interference cancellation. Partition $\boldsymbol{T}_{n}=\left[\begin{array}{llll}\boldsymbol{T}_{n}^{(1)} & \boldsymbol{T}_{n}^{(2)} & \ldots & \boldsymbol{T}_{n}^{(L)}\end{array}\right]$, where $\boldsymbol{T}_{n}^{(l)}$ consists of the additional columns necessary to upgrade the beamformer performance from level $l-1$ to level $l$. This natural ordering is profitably utilized to tradeoff steady state performance for adaptive algorithm convergence rate by dynamically adjusting the number of adaptive weights as suggested in [10].

Remark 4: Both design methods require specification of a set of likely interference scenarios. The performance within this set is guaranteed to satisfy the performance constraint. However, there are no guarantees on the performance for scenarios that are not contained in the set used for design. Hence, the sensitivity of the design to variation between actual and assumed interference scenarios is an important issue. This topic is addressed for the interference output power minimization method in [7] using an argument based on matching the response of the fixed $\left(\boldsymbol{w}_{0}\right)$ and adaptive $\left(\boldsymbol{T}_{n} \boldsymbol{w}_{n}\right)$ GSC branches. The results indicate that the design is relatively insensitive if the set of design matrices $\mathcal{Q}$ is chosen appropriately. Since the analysis and conclusions of [7] are based on response matching, they are independent of the design method and are thus directly applicable to the two methods proposed in this correspondence.

\section{EXAMPLES}

The PD and CMD design procedures are now illustrated using an array employing fifteen sensors arranged in an equally spaced linear geometry with six tap FIR filters in each sensor channel. Hence, there are a total of ninety weights. All signals (desired signal and interferers) are assumed to lie in the normalized frequency band $\left[\frac{3}{5} \pi, \frac{4}{5} \pi\right]$. The desired signal has a direction sine of 0.1 and is uncorrelated with the interferers. Eleven of the available ninety degrees of freedom are used to provide a unit gain and linear phase response in the desired signal direction. The interference spectra are assumed white on $\left[\frac{3}{5} \pi, \frac{4}{5} \pi\right]$.

Partially adaptive beamformers are designed assuming that the direction sines of the interferers are within either $[-0.2,0.025]$ or $[0.175,1]$. For simplicity, we further assume that there are two interferers of power $40 \mathrm{~dB}$ relative to the background white noise level. The goal is to design a partially adaptive beamformer with interference output power within $3 \mathrm{~dB}$ of that provided by a fully adaptive beamformer over all possible combinations of interference directions. Three partially adaptive beamformers are designed using: a single-level PD, a three-level PD, and a three-level CMD (with neighborhood size about 0.15 direction sine).

Partially adaptive beamformer performance is evaluated at 136 different combinations of the two interference direction sines evenly distributed on $[-0.2,0.025]$ and $[0.175,1]$. The performance is summarized in Table I and illustrated graphically in Fig. 2(a) to (c). For comparison purposes, the performance of the minimum interference output power (MIOP) [11] designed partially adaptive beamformer given as an example in [7] is also summarized in Table I and illustrated in Fig. 2(d).

The single-level point design yields a 13-D adaptation space while the three-level point design yields a 12-D adaptation space. This suggests that the multi-level procedure does use the adaptive degrees of freedom more efficiently. The same three-level performance sequence is also used for the constrained minimization design. The result is an 11-D adaptation space; the dimension is further reduced.

The performance losses of all four beamformers designed here are consistently less than $3 \mathrm{~dB}$ as specified. This is contrasted with the performance of the partially adaptive beamformer designed according to the somewhat adhoc region based MIOP approach. Although the adaptation space dimension is 13 , the worst-case loss is greater than 5 
TABLE I

Partially Adaptive Beamformer Performance Summary. (The Ordered Numbers within \{ \} Are the Performance Level Sequences Used in Each Design and \# Denotes Sequence LENGTH.)

\begin{tabular}{ccccccc}
\hline $\begin{array}{l}\text { Design } \\
\text { Method }\end{array}$ & $\begin{array}{c}\text { design level } \\
\delta_{l}(\mathrm{~dB})\end{array}$ & $\begin{array}{c}d i_{m} \\
\left(T_{n}\right)\end{array}$ & $\begin{array}{c}\max \\
\text { loss } \\
(\mathrm{dB})\end{array}$ & $\begin{array}{c}\text { min loss } \\
(\mathrm{dB})\end{array}$ & $\begin{array}{c}\text { ave. } \\
\text { loss } \\
(\mathrm{dB})\end{array}$ & $\begin{array}{c}\text { Performance } \\
\text { Fig. No. }\end{array}$ \\
\hline PD $\quad \#\{-3\}=1$ & 13 & 1.55 & 0.00 & 0.35 & $2(\mathrm{a})$ \\
PD $\#\{-35,-5,-3\}=$ & 12 & 1.81 & 0.00 & 0.25 & $2(\mathrm{~b})$ \\
3 & & & & & \\
CMD $\#\{-35,-5,-3\}=$ & 11 & 1.90 & 0.02 & 0.49 & $2(\mathrm{c})$ \\
MIOP & $\begin{array}{c}3 \\
\text { unspecified }\end{array}$ & 13 & 5.89 & 0.21 & 1.60 & 2(d) \\
\hline
\end{tabular}

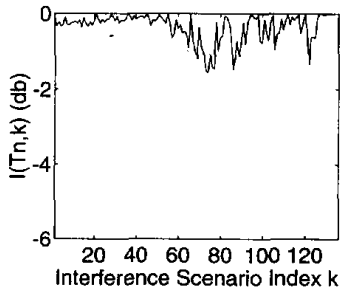

(a)

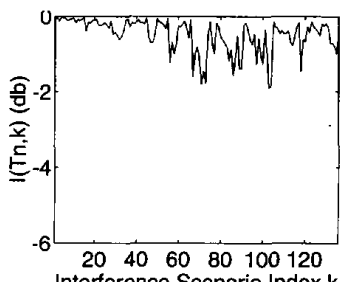

(c)

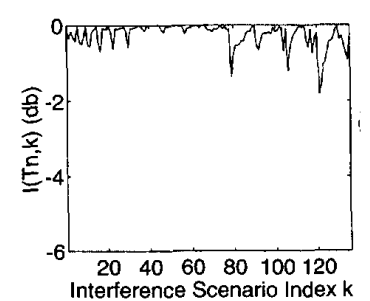

(b)

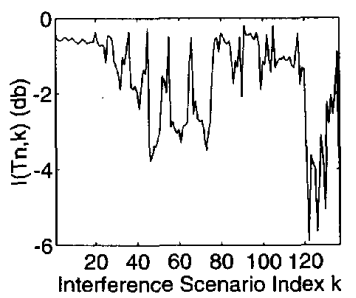

(d)
Fig. 2. Performance loss evaluated at 136 different combinations of interferer directions for the partially adaptive beamformer designed using (a) single-level PD; (b) three-level PD; (c) three-level CMD; (d) MIOP.

dB. The MIOP-designed partially adaptive beamformer also exhibits much greater average performance loss. Therefore, besides being more systematic, the design methods presented here result in better performance while using fewer adaptive degrees of freedom.

\section{REFERENCES}

[1] D. J. Chapman, "Partial adaptivity for the large array" IEEE Trans. Antenna Propagat., vol. AP-24, pp. 685-696, Sept. 1976.

[2] K. A. Byerly and R. A. Roberts, "Output power based partial adaptive array design," in Proc. Twenty-third Asilomar Conf. Signal Syst. Comput., 1989, pp. 576-580, vol. 2.

[3] B. D. Van Veen and C. H. Lee, "Adaptive detection in subspaces," in Proc. Fourth Spectrum Estimation Modeling Workshop, (Rochester, NY), Oct. 1990 , pp. 163-167.

[4] W. F. Gabriel, "Using spectral estimation techniques in adaptive processing antenna systems," IEEE Trans. Antennas Propagat., vol. AP-34, pp. 291-300, Mar. 1986.

[5] L. J. Griffiths and C. W. Jim, "An alternative approach to linearly constrained adaptive beamforming," IEEE Trans. Antennas Propagat. vol. AP-30, pp. 27-34, Jan. 1982.

[6] T. T. Ma and L. J. Griffiths, "A solution space approach to achieving partially adaptive arrays," Proc. IEEE. pp. 2869-2872, Apr. 1988.

[7] B. D. Van Veen, "Minimum variance beamforming," in Adaptive Radar Detection and Estimation (S. Haykin and A. Steinhardt, Eds.) New York: Wiley, 1992, ch. 4.
[8] D. R. Morgan, "Partially adaptive array techniques," IEEE Trans. Antennas Propagat., vol. AP-26, pp. 832-833, Nov. 1978.

[9] N. L. Owsley, "Sonar array processing," in Array Signal Processing (S Haykin, Ed.) Englewood Cliffs: Prentice-Hall, 1985.

[10] T. C. Liu and B. D. Van Veen, "A modular structure for implementation of linearly constrained minimum variance beamformers," IEEE Trans. Signal Processing, vol. 39, pp. 2343-2346, Oct. 1991.

[11] B. D. Van Veen and R. A. Roberts, "Partially adaptive beamformer design via output power minimization," IEEE Trans. Acoust. Speech Signal Processing, vol. ASSP-35, pp. 1524-1532, Nov. 1987.

[12] B. D. Van Veen, "Eigenstructure based partially adaptive array design," IEEE Trans. Antennas Propagat., vol. 36, pp. 357-362, Mar. 1988

[13] _ , "An analysis of several partially adaptive beamformer designs," IEEE Trans. Acoust. Speech Signal Processing, vol. 37, pp. 192-203, Feb. 1989.

[14] _ "Adaptive convergence of linearly constrained beamformers based on the sample covariance matrix," IEEE Trans. Signal Processing, vol. 39, pp. 1470-1473, Jun. 1991.

\section{An Algorithm for Efficient, Unbiased, Equation-Error Infinite Impulse Response Adaptive Filtering}

Carlos E. Davila

Abstract-An algorithm for efficiently adjusting the coefficients of equation-error infinite impluse response (IIR) adaptive filters is described. Unlike the RLS algorithm, the proposed algorithm yields unbiased filter coefficients. Simulations involving the identification of unknown polezero systems demonstrate the algorithm's improved performance over the equation-error RLS algorithm.

\section{INTRODUCTION}

Adaptive infinite impulse response (IIR) filters offer certain advantages over adaptive finite impulse response (FIR) filters, including the need for lower filter orders and the ability to effectively model a wider variety of systems than FIR filters. Correspondingly, adaptive IIR filters have received considerable interest in the signal processing literature over the past several years [1]-[3]. The equation-error adaptive IIR filter output is given by

$$
y(t)=\sum_{m=1}^{N-1} a_{m}(t) d(t-m)+\sum_{m=0}^{M-1} b_{m}(t) x(t-m)
$$

where $d(t)$ is the desired signal. In vector notation, (1) may be written as $y(t)=\theta_{t}^{T} \phi_{t}$, where

$$
\begin{gathered}
\theta_{t}=\left[a_{1}(t) a_{2}(t) \cdots a_{N-1}(t) b_{0}(t) b_{1}(t) \cdots b_{M-1}(t)\right]^{T} \\
\phi_{t}=[d(t-1) d(t-2) \cdots d(t-N+1) x(t) x(t-1) \\
\cdots x(t-M+1)]^{T} .
\end{gathered}
$$

It can be shown that the equation-error adaptive filter coefficient vector generally converges to a biased value. Consider the

Manuscript received December 10, 1990; revised March 3, 1993. The associate editor coordinating the review of this paper and approving it for publication was Prof. V. John Mathews.

The author is with the Electrical Engineering Department, Southern Methodist University, Dallas, TX 75275-0335.

IEEE Log Number 9214179.

Note: Due to a problem in the post production of this correspondence when it originally appeared (This TRANSACTIONS, vol. 42, no. 2, February 1994, pp. 415-419), five of the six tables that were supposed to appear were inadvertently omitted. Therefore, this correspondence is being re-published in its entirety. 